\section{Mechanical thrombectomy in basilar artery occlusion: influence of reperfusion on clinical outcome and impact of the first-line strategy (ADAPT vs stent retriever)}

TO THE READERSHIP: An error appeared in the article by Gory et al. (Gory B, Mazighi M, Blanc R, et al: Mechanical thrombectomy in basilar artery occlusion: influence of reperfusion on clinical outcome and impact of the first-line strategy (ADAPT vs stent retriever). $J$ Neurosurg [epub ahead of print January 12, 2018; DOI: 10.3171/2017.7.JNS171043]).

In the Appendix, the list of ETIS research investigators was incomplete. The updated list is shown here.

Jean-Pierre Decroix, Foch Hospital; Adrien Wang, Foch Hospital; Serge Evrard, Foch Hospital; Maya Tchikviladzé, Foch Hospital; Frederic Bourdain, Foch Hospital; Vadim Afanasiev, Foch Hospital; Loubna Majhadi, Foch Hospital; Bertrand Lapergue, Foch Hospital; Arthuro Consoli, Foch Hospital; Federico Di Maria, Foch Hospital; Oguzhan Coskun, Foch Hospital; Georges Rodesch, Foch Hospital; Delphine Lopez, Foch Hospital; Michel Piotin, Rothschild Foundation; Raphael Blanc, Rothschild Foundation; Hocine Redjem, Rothschild Foundation; Simon Escalard, Rothschild Foundation; Jean-Philippe Desilles, Rothschild Foundation; Gabriele Ciccio, Rothschild Foundation; Stanislas Smajda, Rothschild Foundation; Mikael Mazighi, Rothschild Foundation; Robert Fahed, Rothschild Foundation; Mickael Obadia, Rothschild Foundation; Candice
Sabben, Rothschild Foundation; Guillaume Taylor, Rothschild Foundation; Malek Ben Maacha, Rothschild Foundation; Fernando Pico, Versailles Hospital; Haja Rakotoharinandrasana, Dreux Hospital; Philippe Tassan, Poissy Hospital; Roxanna Poll, Pontoise Hospital; Ovide Corabianu, Aulnay Hospital; Thomas de Broucker, Saint-Denis Hospital; Didier Smadja, Sud Francilien Hospital; Sonia Alamowitch, CHU Tenon; Mickael Obadia, Rothschild Foundation; Olivier Ille, Mantes la Jolie Hospital; Eric Manchon, Gonesse Hospital; Pierre-Yves Garcia, CHU Amiens; Roberto Riva, Hospices Civils de Lyon; Paul-Emile Labeyrie, Hospices Civils de Lyon; Norbert Nighoghossian, Hospices Civils de Lyon; Benjamin Gory, Hospices Civils de Lyon; Frédéric Philippeau, Bourg-en-Bresse Hospital; Serkan Cakmak, Nord Ouest Hospital; Karine Blanc-Lasserre, Valence Hospital; and Anne-Evelyne Vallet, Vienne Hospital.

The article has been corrected online as of May 25, 2018.

Benjamin Gory, MD, PhD

INSERM U947, University Hospital of Nancy, University of Lorraine, Nancy, France

Bertrand Lapergue, MD, PhD

Stroke Center, Foch Hospital, Université de Versailles Saint-Quentin-en-Yvelines, Suresnes, France

CORRESPONDING ARTICLE See pp 1482-1491.

INCLUDE WHEN CITING

Published online May 25, 2018; DOI: 10.3171/2018.3.JNS171043a.

CAANS 2018, except where prohibited by US copyright law 